\title{
Thermal characterization of a new effective building material based on clay and olive waste
}

\author{
Lamrani Mohamed $^{1}$, Khalfaoui Mohamed ${ }^{1}$, Laaroussi Najma ${ }^{2}$ and Khabbazi Abdelhamid ${ }^{2}$ \\ ${ }^{1}$ Mohammed V University in Rabat, EST de Salé, LASTIMI, Salé, Maroc \\ ${ }^{2}$ Mohammed V University in Rabat, EST de Salé, MEAT-EMDD, Salé, Maroc
}

\begin{abstract}
The influence of thermophysical properties of wall materials on energy performance and comfort in traditional building was investigated. The clay is the most commonly used sustainable building material. The study looked at the effects of the addition of pomace olive on the thermophysical properties of clay bricks to improve the energy efficiency of this ecological material. An experimental measurement of thermal properties of clay mixed with pomace olive was carried out by using the transient and steady state hot-plate and flash methods. The experimental methods are applied to measure the thermal properties of the composite material. The estimation of these thermal characteristics is based on a one dimensional model and the experimental errors are found less than $3 \%$. The composite samples were prepared with different granular classes and mass fractions of the pomace olive in the mixture. The results show that the density of the new material was not substantially influenced by the size of the pomace olive. However, the thermal conductivity and diffusivity decrease from $0.65 \mathrm{~W} \cdot \mathrm{m}^{-1} \cdot \mathrm{K}^{-1}$ and $4.21 \times 10^{-7} \mathrm{~m}^{2} \cdot \mathrm{s}^{-1}$ to $0.29 \mathrm{~W} \cdot \mathrm{m}^{-1} \cdot \mathrm{K}^{-1}$ and $2.47 \times 10^{-7} \mathrm{~m}^{2} \cdot \mathrm{s}^{-1}$, respectively, according to the variation of the volume fraction of pomace olive from 0 (pure clay) to $71 \%$ showing that the olive pomace can be used as effective secondary raw materials in the making of clay bricks.

Key words: Ecological material, building efficiency, insulation, thermophysical characterization.
\end{abstract}

\section{Introduction}

Actuellement, la valorisation des matériaux locaux utilisés dans le secteur du bâtiment et l'économie d'énergie constituent un champ de recherche très dynamique au Maroc. Plusieurs études ont été menées sur des matériaux locaux tels que l'argile et le plâtre consolidés avec des produits de nature végétale ou animale. Cherki et al. [1] ont étudié les propriétés thermiques d'un matériau isolant à base du liège et du plâtre. L'effet des fibres d'alfa sur les caractéristiques thermophysiques de l'argile a été mis en évidence par Elhamdouni et al. [2]. Mounir et al. [3] ont réalisé une étude expérimentale des propriétés thermiques de l'argile consolidée avec du liège utilisant la méthode du plan chaud en régime permanent et transitoire et la méthode flash. L'efficacité énergétique d'un matériau composite à base d'argile et de paille a été montrée par El Azhary et al. [4]. Les propriétés thermiques des briques en terre cuite ont été mesurées en utilisant différentes techniques de métrologie thermique par Laaroussi et al. [5-6].

Notre étude expérimentale est basée sur la caractérisation d'un matériau composite réalisé par l'incorporation des résidus des grignons d'olive consolidés avec de l'argile qui constitue l'élément de base du pisé au sud marocain.

Le but de ce travail est consacré à mettre en évidence l'influence de la taille et des proportions occupées par les grignons dans le mélange sur les propriétés du matériau testé. L'identification de ces propriétés thermophysiques est faite à l'aide de la méthode du plan chaud asymétrique en régimes permanent et transitoire développée par Jannot et al. [7] pour la mesure de la conductivité et l'effusivité thermiques et la méthode Flash pour la mesure de la diffusivité thermique.

\section{Matériaux utilisés}

Argile : L'argile utilisée dans cette étude est extraite de la région d'Errachidia au sud marocain.

Grignon d'olive : Le grignon d'olive est le rejet d'une huilerie traditionnelle de la région. Il est séché pendant quelques jours à l'air libre. Les résultats d'analyse chimique sont présentés dans le tableau 1.

Tableau 1. Analyse chimique du grignon d'olive.

\begin{tabular}{|c|c|c|c|c|}
\hline Cellulose & $\begin{array}{c}\text { Hémi- } \\
\text { cellulose }\end{array}$ & Lignine & $\begin{array}{c}\text { Matière } \\
\text { grasse }\end{array}$ & Cendres \\
\hline $34.25 \%$ & $12.40 \%$ & $23.36 \%$ & $4.33 \%$ & $3.01 \%$ \\
\hline
\end{tabular}

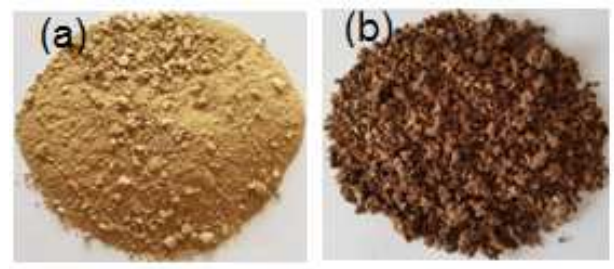

Fig. 1. (a) Argile, (b) Grignons d'olive. 


\section{Préparation des échantillons}

La préparation des échantillons consiste à répartir les grignons d'olive en classes avec différentes tailles par un procédé de tamisage normalisé et à préparer la pâte d'argile avec un taux de gâchage massique w/g $=0.25$. Les échantillons fabriqués ont des épaisseurs de $20 \mathrm{~mm}$ et sont élaborés selon deux approches différentes :

1- Des échantillons dont la variable est la taille des grignons d'olive. Trois échantillons correspondants à cinq classes avec différentes tailles $\left(\mathrm{d}_{1}-\mathrm{D}_{1}=0.63-1.25 \mathrm{~mm}\right.$, $\mathrm{d}_{2}-\mathrm{D}_{2}=1.25-2.5 \mathrm{~mm}, \mathrm{~d}_{3}-\mathrm{D}_{3}=2.5-5 \mathrm{~mm}, \mathrm{~d}_{4}-\mathrm{D}_{4}=5-8 \mathrm{~mm}$ et $\mathrm{d}_{5}-\mathrm{D}_{5}=8-12.5 \mathrm{~mm}$ ) ont été élaborés dans des moules identiques dont les dimensions sont $100 \times 100 \times 20 \mathrm{~mm}^{3}$. Le moule est entièrement rempli par un volume des grignons d'olive correspondant à une teneur $\mathrm{X}=100 \%$, ensuite la pâte d'argile est ajoutée afin d'occuper l'espace inter-granulaire. La distribution des grains dans le mélange est assurée par une bétonnière pour homogénéiser le matériau composite.

2- La préparation d'échantillons dont la variable est la teneur, $\mathrm{X}$, des grignons d'olive dans le mélange pour la classe $\mathrm{d}_{2}-\mathrm{D}_{2}=1.25-2.5 \mathrm{~mm}$. On rempli le volume apparent par des fractions volumiques des grignons décroissantes variantes de $100 \%, 80 \%, 60 \%, 40 \%, 20 \%$ à $\mathrm{X}=0 \%$ représentant un échantillon d'argile brute sans additifs afin de comparer la variation de ses propriétés thermiques avec ceux du matériau composite. La figure 2 représente l'aspect des échantillons fabriqués avec différentes tailles des grignons d'olive. Les échantillons ont été séchés à l'étuve pour éliminer l'humidité présente dans les pores. Ensuite, leurs masses ont été mesurées puis rangées dans des sacs en plastique.

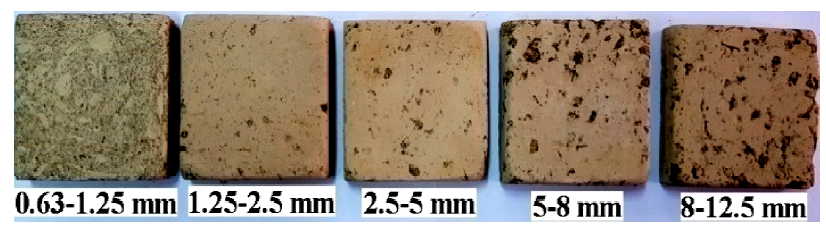

Fig. 2. Echantillons argile-grignons d'olive avec différentes tailles étudiées.

\section{Approche théorique de mesure}

\subsection{Méthode du Plan chaud asymétrique}

La méthode du plan chaud asymétrique en régime permanent permet d'identifier la conductivité thermique d'un matériau compris entre deux plans isothermes "Figure 3".

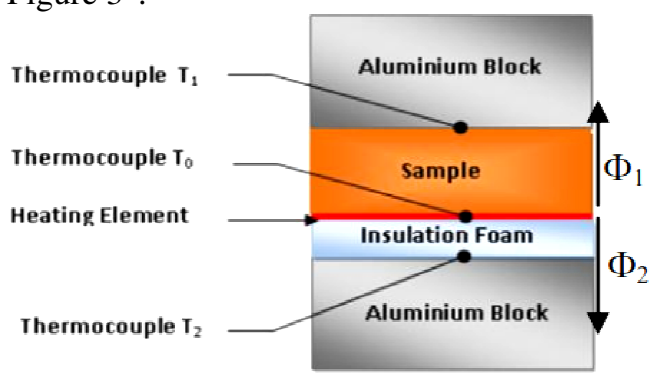

Fig. 3. Dispositif expérimental de la méthode Plan chaud asymétrique en régime permanent.
L'échantillon étudié est placé sur un élément chauffant, sous lequel est placée une mousse isolante en polyéthylène ayant les dimensions de $10 \times 100 \times 100 \mathrm{~mm}^{3}$ et de conductivité $0,04 \mathrm{~W} \cdot \mathrm{m}^{-1} \cdot \mathrm{K}^{-1}$, pour que la majorité $\mathrm{du}$ flux de chaleur, émis par l'élément chauffant, passe à travers l'échantillon. Cet ensemble est placé ensuite entre deux blocs d'aluminium de dimensions $50 \times 100 \times$ $100 \mathrm{~mm}^{3}$ qui ont une grande conductivité et jouent le rôle de plaques isothermes pour que le système atteint l'équilibre thermique le plus vite possible.

La mesure des températures est faite à l'aide des thermocouples mesurant $T_{0}, T_{1}$ et $T_{2}$ placés aux centres des faces de l'échantillon et de la mousse isolante. La conductivité thermique $\lambda_{1}$ de l'échantillon, une fois que le système ait atteint le régime permanent, est donnée par l'équation (1) :

$\lambda_{1}=\frac{e_{1}}{T_{0}-T_{1}}\left[\frac{U^{2}}{R_{\Omega} . S}-\frac{\lambda_{2}}{e_{2}}\left(T_{0}-T_{2}\right)\right]$

Où $\mathrm{e}_{1}$ est l'épaisseur de l'échantillon ; $\lambda_{2}=0.04 \mathrm{~W} \cdot \mathrm{m}^{-1} \mathrm{~K}^{-1}$ et $\mathrm{e}_{2}=10 \mathrm{~mm}$ sont successivement la conductivité thermique et l'épaisseur de la mousse isolante ; U est la tension imposée aux bornes de l'élément chauffant de résistance électrique $R_{\Omega}=40 \Omega$ et de surface $S=0.0105 \mathrm{~m}^{2}$.

L'étalonnage du système expérimental a été réalisé à l'aide d'un échantillon de référence à conductivité connue.

\subsection{Méthode Flash}

La caractérisation de la diffusivité thermique est effectuée par la méthode Flash par résolution de l'équation de la chaleur dans l'espace de Laplace en utilisant le modèle quadripolaire [8].

Une impulsion thermique $\emptyset_{0}\left(\mathrm{~W} . \mathrm{m}^{-2}\right)$ de courte durée a été envoyée sur la face supérieure de l'échantillon parfaitement isolé à la périphérie. Les pertes de chaleur des deux faces de l'échantillon $\left(100 \times 100 \times 20 \mathrm{~mm}^{3}\right)$ sont représentées par le même coefficient d'échange convectif h. L'élévation de température est mesurée par un thermocouple placé au centre de la face non irradiée "Figure 4".

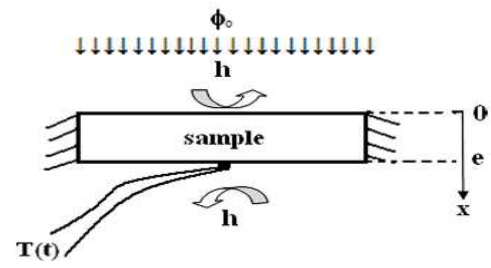

Fig. 4. Principe de la méthode Flash.

L'élévation de la température dans l'espace de Laplace est donnée par l'expression suivante :

$$
\theta(p)=\frac{\frac{\emptyset_{0}\left(1-e^{-\tau \cdot p}\right)}{p}}{h^{2} \frac{\sinh (k e)}{\lambda k}+2 h \cosh (k e)+\lambda k \cdot \sinh (k e)}
$$

$\tau$ est la durée de l'impulsion thermique, $=\sqrt{\frac{\mathrm{p}}{a}}, \mathrm{p}$ est le paramètre de Laplace et a est la diffusivité thermique.

$\lambda$ et e sont succéssivement la conductivité thermique et l'épaisseur de l'échantillon. 
Après l'inversion numérique de cette expression par l'algorithme de De Hoog [9], on obtient l'expression théorique de la température. L'algorithme de LevenbergMarquardt [10-11] est utilisé pour l'estimation des paramètres $\mathrm{a}, \emptyset_{0}$ et $\mathrm{h}$ par minimisation de l'erreur quadratique entre le thermogramme expérimental représentant l'évolution de la température de la face arrière de l'échantillon et l'expression théorique de la température.

\subsection{Méthode du Plan chaud asymétrique en régime transitoire}

La méthode du plan chaud asymétrique en régime transitoire permet d'identifier l'effusivité thermique de l'échantillon "Figure 5". L'échantillon est posé sur un élément chauffant de même surface $\left(100 \times 100 \mathrm{~mm}^{2}\right)$, un thermocouple de type $\mathrm{K}$ est installé au centre de la face inférieure de l'élément chauffant. Le dispositif est placé entre deux blocs de polystyrène ayant chacun une épaisseur de $50 \mathrm{~mm}$. L'ensemble est ensuite placé entre deux blocs d'aluminium d'épaisseur $40 \mathrm{~mm}$ chacun. Un échelon de flux de chaleur est émis par l'élément chauffant et l'élévation de température $\mathrm{T}(\mathrm{t})$ au centre de l'échantillon est enregistrée.

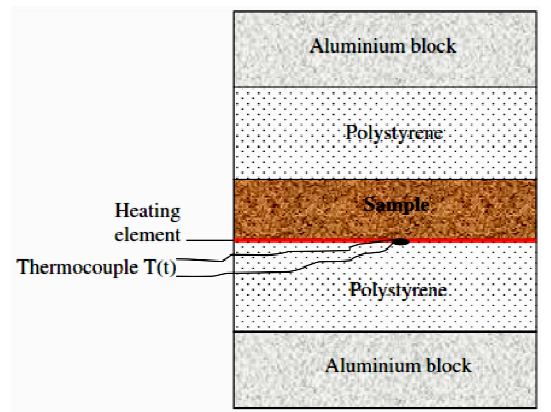

Fig. 5. Dispositif expérimental de la méthode Plan chaud asymétrique en régime transitoire

Le système est modélisé avec l'hypothèse d'un transfert de chaleur unidirectionnel (1D) au centre de l'échantillon. Compte tenu de la faible valeur du flux de chaleur atteignant les blocs d'aluminium à travers le polystyrène et leurs grandes capacités thermiques, leurs températures sont supposées égales et constantes. En appliquant le formalisme des quadripôles sur le modèle représenté par la figure 3 , on peut écrire :

$$
\begin{gathered}
{\left[\begin{array}{c}
\theta \\
\Phi_{01}
\end{array}\right]=\left[\begin{array}{cc}
1 & 0 \\
c_{h} & 1
\end{array}\right]\left[\begin{array}{cc}
1 & R_{c} \\
0 & 1
\end{array}\right]\left[\begin{array}{ll}
A & B \\
C & D
\end{array}\right]\left[\begin{array}{ll}
A_{i} & B_{i} \\
C_{i} & D_{i}
\end{array}\right]\left[\begin{array}{c}
0 \\
\Phi_{1}
\end{array}\right]} \\
=\left[\begin{array}{ll}
A_{1} & B_{1} \\
C_{1} & D_{1}
\end{array}\right]\left[\begin{array}{c}
0 \\
\Phi_{1}
\end{array}\right] \\
{\left[\begin{array}{c}
\theta \\
\Phi_{02}
\end{array}\right]=\left[\begin{array}{ll}
A_{i} & B_{i} \\
C_{i} & D_{i}
\end{array}\right]\left[\begin{array}{c}
0 \\
\Phi_{2}
\end{array}\right]} \\
\Phi_{0}=\frac{\emptyset}{p}=\Phi_{01}+\Phi_{02}
\end{gathered}
$$

$\theta$ est la transformée de Laplace de l'élévation de température $\mathrm{T}(\mathrm{t}) ; \Phi_{01}$ est la transformée de Laplace de la densité de flux de chaleur dissipée par l'élément chauffant vers l'échantillon (vers le haut); $\Phi_{02}$ est la transformée de Laplace de la densité de flux de chaleur dissipée par l'élément chauffant vers le polystyrène (vers le bas) ; $\Phi_{0}$ est la transformée de Laplace de la densité de flux de chaleur totale produite par l'élément chauffant ; Ø est la densité de flux de chaleur totale produite par l'élément chauffant et $C_{h}$ sa capacité thermique $\left(C_{h}=\rho_{h}\right.$ $\left.c_{\mathrm{h}} \mathrm{e}_{\mathrm{h}}\right) ; \Phi_{l}$ est la transformée de Laplace de la densité de flux de chaleur qui atteint le bloc d'aluminium du haut; $\Phi_{2}$ est la transformée de Laplace de la densité de flux de chaleur qui atteint le bloc d'aluminium du bas. avec :

$$
\begin{aligned}
& A=D=\cosh \left(\frac{\rho c}{E} e \sqrt{p}\right) ; B=\frac{\sinh \left(\frac{\rho c}{E} e \sqrt{p}\right)}{E \sqrt{p}} ; \\
& C=E \sqrt{p} \sinh \left(\frac{\rho c}{E} e \sqrt{p}\right) \\
& A_{i}=D_{i}=\cosh \left(\sqrt{\frac{p}{a_{i}}} e_{i}\right) ; B_{i}=\frac{\sinh \left(\sqrt{\frac{p}{a_{i}}} e_{i}\right)}{\lambda_{i} \sqrt{\frac{p}{a_{i}}}} ; \\
& C_{i}=\lambda_{i} \sqrt{\frac{p}{a_{i}}} \sinh \left(\sqrt{\frac{p}{a_{i}}} e_{i}\right)
\end{aligned}
$$

$E$ est l'effusivité thermique de l'échantillon $E=\sqrt{\lambda \rho c}$; $\rho c$ est la capacité thermique volumique de l'échantillon; e est l'épaisseur de l'échantillon; $\lambda_{\mathrm{i}}, \mathrm{a}_{\mathrm{i}}$ et $\mathrm{e}_{\mathrm{i}}$ sont respectivement la conductivité thermique, la diffusivité thermique et l'épaisseur du polystyrène.

En combinant ces cinq dernières équations, on en déduit la valeur de $\theta$ par la relation :

$$
\theta(p)=\frac{\Phi_{0}(p)}{\frac{D_{1}}{B_{1}}+\frac{D_{i}}{B_{i}}}
$$

Le principe de cette méthode est d'estimer les valeurs des paramètres $\left(E, \rho c, R_{c}, C_{h}\right)$ en minimisant l'erreur quadratique $\Psi=\sum_{\mathrm{j}=0}^{\mathrm{N}}\left[\mathrm{T}_{\exp }\left(\mathrm{t}_{\mathrm{j}}\right)-\mathrm{T}_{\bmod }\left(\mathrm{t}_{\mathrm{j}}\right)\right]^{2}$ entre les points expérimentaux et les valeurs théoriques calculées par la transformée de Laplace inverse de la relation (8) en utilisant l'algorithme de Levenbeg-Marquart [10-11]. La transformée de Laplace inverse de la relation (8) est réalisée en utilisant l'algorithme de De Hoog [9].

\section{Résultats et discussions}

Pour chaque échantillon, trois tests ont été réalisés pour tenir compte de l'erreur de mesure et pour que les résultats de caractérisation obtenus soit statistiquement validés. La moyenne arithmétique de ces trois essais est adoptée comme résultat final de caractérisation pour chaque échantillon testé.

\subsection{Masse volumique des échantillons}

Connaissant les dimensions et les masses sèches des échantillons, la masse volumique de chaque échantillon a été calculée "Tableau 2".

La loi des mélanges pour un milieu à deux composants a été utilisée pour déduire la fraction volumique, notée $y$, des grignons dans le mélange.

$$
\rho_{G o-a r g}=y \cdot \rho_{G o}+(1-y) \rho_{\text {arg }}
$$

avec: $\rho_{\mathrm{Go}-\mathrm{arg}}, \rho_{\mathrm{arg}}$ et $\rho_{\mathrm{Go}}$ sont respectivement les masses volumiques apparentes du matériau composite, de l'argile et des grignons d'olive. 
Tableau 2. Masse volumique apparente moyenne et fraction volumique des grignons d'olive des échantillons fabriqués.

\begin{tabular}{|c|c|c|c|c|c|}
\hline $\mathbf{N}^{\circ}$ & Echantillon & $\mathrm{X}(\%)$ & $\begin{array}{c}\rho_{\text {moy }} \\
\left(\mathrm{kg} \cdot \mathrm{m}^{-3}\right)\end{array}$ & $\left.\frac{\Delta \rho}{\rho_{\text {moy }}} \%\right)$ & $\mathrm{y}$ \\
\hline 1 & Go-ag $0.63-1.25 \mathrm{~mm}$ & \multirow{5}{*}{100} & 1138.55 & 0,088 & 0.502 \\
\hline 2 & Go-ag $1.25-2.5 \mathrm{~mm}$ & & 1142.42 & 0.121 & 0.499 \\
\hline 3 & Go-arg $2.5-5 \mathrm{~mm}$ & & 1139.14 & 0.079 & 0.502 \\
\hline 4 & Go-arg 5-8 mm & & 1141.55 & 0.102 & 0.500 \\
\hline 5 & Go-arg 8-12.5 mm & & 1140.19 & 0.192 & 0.501 \\
\hline 6 & Go-arg $1.25-2.5 \mathrm{~mm}$ & 80 & 1239.17 & 0.206 & 0.423 \\
\hline 7 & Go-arg $1.25-2.5 \mathrm{~mm}$ & 60 & 1398.3 & 0.219 & 0.298 \\
\hline 8 & Go-arg $1.25-2.5 \mathrm{~mm}$ & 40 & 1553.97 & 0.148 & 0.176 \\
\hline 9 & Go-arg 1.25-2.5mm & 20 & 1642.59 & 0.147 & 0.107 \\
\hline 10 & Argile & 0 & 1778.68 & 0.099 & ----- \\
\hline & Grignons d'olive & & 504 & ----- & ------ \\
\hline
\end{tabular}

Nous constatons que la masse volumique apparente ne varie pratiquement pas en fonction de la taille. Cependant, plus la teneur en grignons augmente dans mélange plus le composite devient de plus en plus léger.

\subsection{Conductivité thermique}

La conductivité thermique des échantillons mesurée par la méthode du Plan chaud asymétrique en régime permanent est représentée dans le tableau 3, l'erreur moyenne issue de l'expérimentation, calculée par la formule $\frac{\Delta \lambda}{\lambda_{\text {moy }}}$, ne dépasse pas $3 \%$.

Tableau 3. Résultats de caractérisation issus des trois méthodes expérimentales utilisées

\begin{tabular}{|c|c|c|c|c|c|}
\hline $\mathbf{N}^{\circ}$ & $\begin{array}{c}\text { Echantillon } \\
\text { Go-arg }\end{array}$ & $\begin{array}{c}X \\
(\%)\end{array}$ & $\begin{array}{c}\lambda \\
\left(\mathrm{W} \cdot \mathrm{m}^{-1} \cdot \mathrm{K}^{-1}\right)\end{array}$ & $\begin{array}{l}\mathrm{a} \times 10^{-7} \\
\left(\mathrm{~m}^{2} \cdot \mathrm{s}^{-1}\right)\end{array}$ & $\left(\begin{array}{c}\mathrm{E} \\
\left(\mathrm{W} \cdot \mathrm{s}^{1 / 2} \cdot \mathrm{m}^{-2} .\right. \\
\left.\mathrm{K}^{-1}\right)\end{array}\right.$ \\
\hline 1 & $0.63-1.25 \mathrm{~mm}$ & \multirow{5}{*}{100} & 0.298 & 2.47 & 595.56 \\
\hline 2 & $1.25-2.5 \mathrm{~mm}$ & & 0.296 & 2.49 & 594.59 \\
\hline 3 & $2.5-5 \mathrm{~mm}$ & & 0.297 & 2.48 & 594.88 \\
\hline 4 & $5-8 \mathrm{~mm}$ & & 0.296 & 2.49 & 594.42 \\
\hline 5 & $8-12.5 \mathrm{~mm}$ & & 0.298 & 2.50 & 596.10 \\
\hline 6 & $1.25-2.5 \mathrm{~mm}$ & 80 & 0.351 & 2.91 & 653.85 \\
\hline 7 & $1.25-2.5 \mathrm{~mm}$ & 60 & 0.401 & 3.15 & 715.28 \\
\hline 8 & $1.25-2.5 \mathrm{~mm}$ & 40 & 0.463 & 3.31 & 805.58 \\
\hline 9 & $1.25-2.5 \mathrm{~mm}$ & 20 & 0.553 & 3.75 & 904.07 \\
\hline 10 & Argile & 0 & 0.651 & 4.21 & 1001.12 \\
\hline \multicolumn{3}{|c|}{ Erreur maximale } & $2.37 \%$ & $2.41 \%$ & $0.86 \%$ \\
\hline
\end{tabular}

$\lambda$ : Conductivité thermique par la méthode du Plan chaud asymétrique en régime permanent.

a: Diffusivité thermique par la méthode Flash.

$\boldsymbol{E}$ : Effusivité thermique par la méthode Plan chaud asymétrique en régime transitoire.
Nous constatons l'influence de l'augmentation de la proportion volumique des grignons d'olive sur la conductivité thermique du matériau, ce qui montre que plus le matériau est léger plus sa qualité d'isolation thermique est améliorée.

\subsection{Diffusivité thermique}

\subsubsection{Résultats de mesure}

La figure 6 illustre le résultat de minimisation des deux thermogrammes expérimental et théorique avec leurs résidus pour l'échantillon de classe $1.25-2.5 \mathrm{~mm}$.

La courbe théorique reste en très bon accord avec les points expérimentaux jusqu'au $\mathrm{t}=1400 \mathrm{~s}$, ceci montre la validité du modèle $1 \mathrm{D}$ au centre de l'échantillon. Cette constatation est validée par l'observation des résidus qui restent faibles et plats le long de l'expérience.

Le tableau 3 regroupe aussi les résultats de la diffusivité thermique moyenne obtenus pour chaque échantillon. L'erreur de mesure moyenne $\left(\frac{\Delta a}{a_{m o y}}\right)$ correspondant à la diffusivité ne dépasse pas $3 \%$. Cela montre que le dispositif expérimental utilisé donne une bonne reproductibilité des mesures.

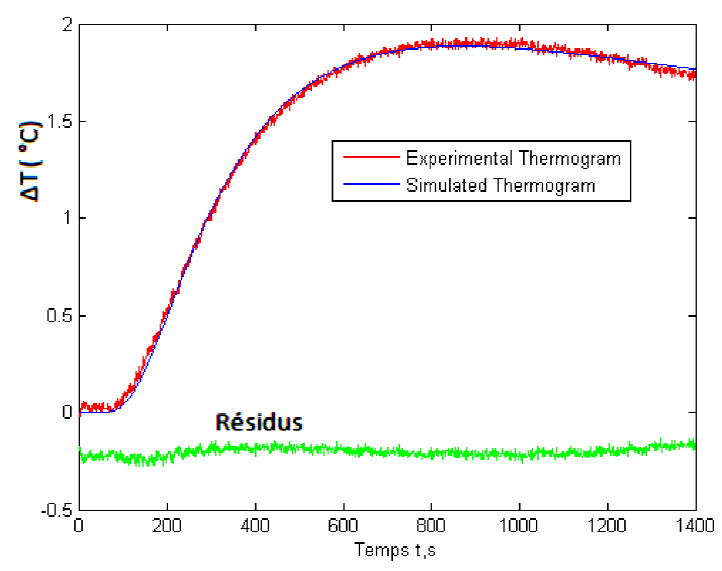

Fig. 6. Minimisation par le modèle complet de la méthode Flash pour l'échantillon de classe $1.25-2.5 \mathrm{~mm}$

\subsubsection{Analyse de la sensibilité}

La figure 7 montre l'allure des courbes des sensibilités réduites $\left(\mathrm{a} \frac{\partial \mathrm{T}}{\partial \mathrm{a}} ; \emptyset_{0} \frac{\partial \mathrm{T}}{\partial \emptyset_{0}} ; \mathrm{h} \frac{\partial \mathrm{T}}{\partial \mathrm{h}}\right)$ relatives aux trois paramètres d'identification. La sensibilité d'un paramètre est le degré d'influence qui nous informe sur la fiabilité des résultats obtenus.

L'analyse des courbes des sensibilités pour les trois paramètres $\left(\mathrm{a}, \emptyset_{0}, h\right)$ à estimer par la méthode Flash montre que la diffusivité peut être identifiée à $\mathrm{t}=300 \mathrm{~s}$ car sa sensibilité est élevée. Pour h et $\emptyset_{0}$, ils sont estimés à des temps plus longs. Le calcul des sensibilités réduites pour chaque paramètre a été programmé sous MATLAB.

L'analyse du thermogramme réalisé sur cet exemple est similaire aux thermogrammes trouvés pour les autres séries d'échantillons. 


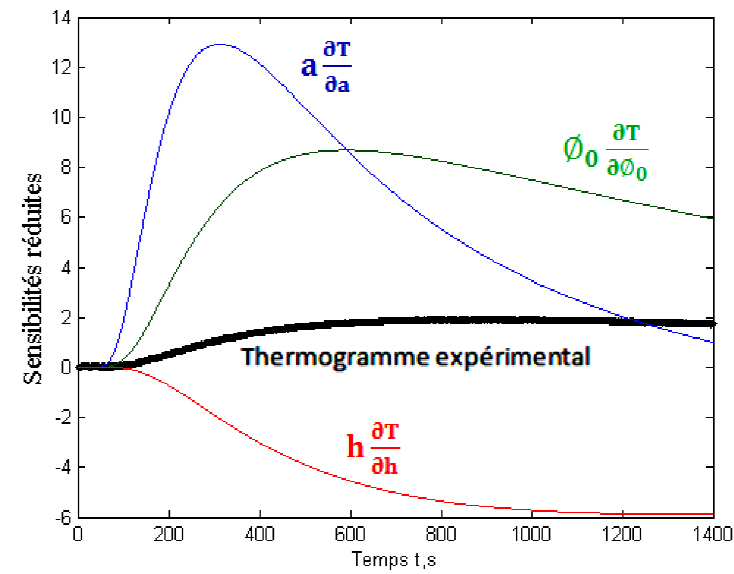

Fig. 7. Courbes des sensibilités réduites des trois paramètres à estimer de l'échantillon de classe 1.25-2.5 mm

\subsection{Effusivité thermique}

La méthode du plan chaud asymétrique transitoire a été appliquée aux échantillons fabriqués du composite argilegrignons d'olive. Pour chaque thermogramme obtenu, l'algorithme de Levenberg-Marquart est appliqué pour identifier les paramètres $\left(\mathrm{E}, \rho \mathrm{c}, \mathrm{Rc}, \mathrm{C}_{\mathrm{h}}\right)$ en minimisant l'écart quadratique entre la courbe expérimentale et la courbe théorique simulée.

La figure 8 montre les courbes expérimentale et simulée après minimisation, avec les résidus correspondants, pour l'un des échantillons de classe granulaire $1.25-2.5 \mathrm{~mm}$.

La figure 9 illustre le tracé des courbes des sensibilités réduites $\left(\mathrm{E} \frac{\partial \mathrm{T}}{\partial \mathrm{E}} ;(\rho \mathrm{c}) \frac{\partial \mathrm{T}}{\partial(\rho \mathrm{c})} ; \mathrm{Rc} \frac{\partial \mathrm{T}}{\partial \mathrm{Rc}}\right.$ et $\left.\mathrm{C}_{\mathrm{h}} \frac{\partial \mathrm{T}}{\partial \mathrm{C}_{\mathrm{h}}}\right)$ relatives aux paramètres d'identification.

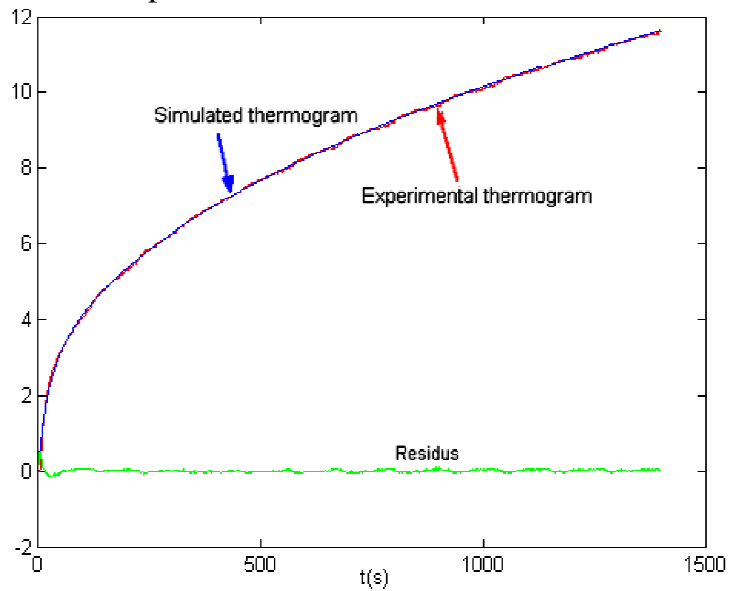

Fig. 8. Minimisation par la méthode Plan chaud transitoire pour l'échantillon de classe $1.25-2.5 \mathrm{~mm}$

D'après la figure 8 , on peut constater que le modèle théorique simulé est en très bon accord avec la courbe expérimental et que le modèle $1 \mathrm{D}$ reste valable jusqu'au $\mathrm{t}=1400 \mathrm{~s}$. Les paramètres $\mathrm{E}$ et $\rho \mathrm{c}$ à estimer donnent des valeurs fiables puisque leurs sensibilités sont élevées.

Le Tableau 3 regroupe les résultats d'identification de l'effusivité thermique des échantillons par la méthode du plan chaud transitoire avec une erreur moyenne $\frac{\Delta E}{E_{m o y}}$ qui ne dépasse pas $1 \%$.
On peut noter que l'éffusivité ne varie pratiquement pas en fonction de la taille des grignons d'olive et décroit significativement en fonction de la teneur en grignons.

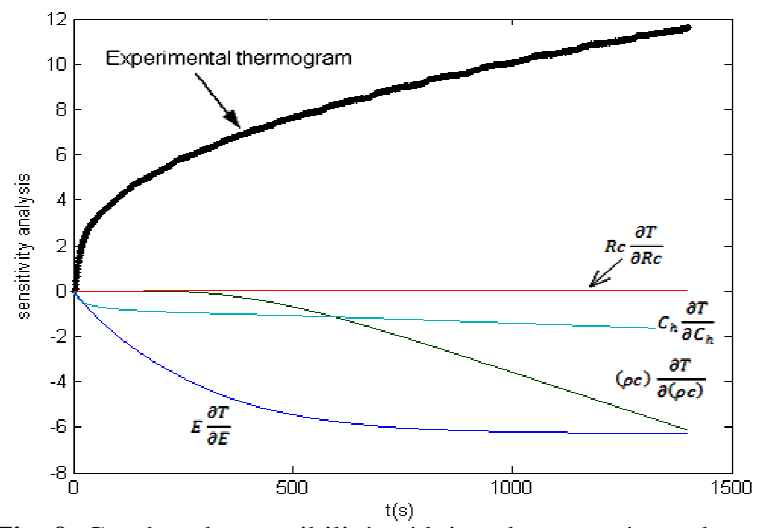

Fig. 9. Courbes des sensibilités réduites des paramètres de l'échantillon de classe $1.25-2.5 \mathrm{~mm}$

Les erreurs de mesure relevées ne dépassent pas $1 \%$ pour toutes les mesures effectuées, ce qui confirme la fiabilité et la robustesse de la méthode adoptée pour la caractérisation du matériau étudié.

\section{Intérêt pratique du composite étudié}

On considère deux murs composés par le composite argile-grignons d'olive et par l'argile brute sans grignons avec la même épaisseur et soumis au même gradient de température en régime permanent ce qui nous a permis de déduire le ratio des deux flux de chaleur traversant ces deux murs en fonction de leur conductivité thermique :

$\frac{\emptyset_{\mathrm{Go}-\mathrm{arg}}}{\emptyset_{\mathrm{arg}}}=\frac{\lambda_{\mathrm{Go}-\mathrm{arg}}}{\lambda_{\mathrm{arg}}}$

En se basant sur les résultats expérimentaux obtenus, on peut calculer le facteur de légèreté et le gain énergétique direct par transmission de chaleur:

Gain_énergétique $=100 \times\left(1-\frac{\emptyset_{\mathrm{Go}-\mathrm{arg}}}{\emptyset_{\mathrm{arg}}}\right)$

Le facteur de légèreté $=100 \times\left(1-\frac{\rho_{\mathrm{Go}-\mathrm{arg}}}{\rho_{\mathrm{arg}}}\right)$

Tableau 4. Gain énergétique et facteur de légèreté pour les différents échantillons par rapport à l'argile brute sans grignons.

\begin{tabular}{|c|c|c|c|c|}
\hline $\mathbf{N}^{\circ}$ & Echantillon & $\mathrm{X}(\%)$ & $\begin{array}{l}\text { Gain } \\
\text { énergétique } \\
(\%)\end{array}$ & $\begin{array}{c}\begin{array}{c}\text { Facteur } \\
\text { de } \\
\text { légèreté } \\
(\%)\end{array} \\
\end{array}$ \\
\hline 1 & Go-arg $0.63-1.25 \mathrm{~mm}$ & \multirow{5}{*}{100} & 54.22 & 35.99 \\
\hline 2 & Go-arg $1.25-2.5 \mathrm{~mm}$ & & 54.53 & 35.77 \\
\hline 3 & Go-arg 2.5-5 mm & & 54.38 & 35.96 \\
\hline 4 & Go-arg 5-8 mm & & 54.53 & 35.82 \\
\hline 5 & Go-arg 8-12.5 mm & & 54.22 & 35.90 \\
\hline 6 & Go-arg $1.25-2.5 \mathrm{~mm}$ & 80 & 46.08 & 30.33 \\
\hline 7 & Go-arg $1.25-2.5 \mathrm{~mm}$ & 60 & 38.40 & 21.39 \\
\hline 8 & Go-arg $1.25-2.5 \mathrm{~mm}$ & 40 & 28.88 & 12.63 \\
\hline 9 & Go-arg $1.25-2.5 \mathrm{~mm}$ & 20 & 15.05 & 7.65 \\
\hline
\end{tabular}


Le tableau 4 regroupe les résultats obtenus de ces deux paramètres pour les différents échantillons par rapport à l'argile brute sans grignons.

Ces résultats montrent que l'incorporation des grignons d'olive dans l'argile permet d'avoir un matériau qui nous permet d'avoir un gain énergétique de $54 \%$ environ avec une légèreté de $36 \%$ que l'argile brute, ce qui améliorera le confort intérieur des locaux et réduira la facture énergétique de conditionnement d'air et contribuera par conséquent à l'amélioration de l'efficacité énergétique dans le bâtiment traditionnel en pisé.

\section{Conclusions}

S'agissant d'un matériau local, naturel, abondant et à faible coût, le grignon d'olive améliore la qualité d'isolation thermique de l'argile et offre une meilleure efficacité énergétique. Le composite ainsi développé peut constituer une alternative intéressante aux matériaux traditionnellement utilisés en construction dans les régions rurales du sud marocain.

Les propriétés thermophysiques du matériau composite sont nettement améliorées par l'ajout progressif des additifs proposés. Ainsi, la teneur en grignons influe positivement sur les performances énergétiques de l'argile en offrant une légèreté de $36 \%$ et un gain énergétique de $54 \%$ environ.

Cependant, les propriétés thermiques du matériau ne constituent pas le seul critère de choix pour la construction, il faut également tenir compte de ses propriétés mécaniques qui constituent un paramètre important pour la stabilité du bâtiment.

\section{Références}

1. A. Cherki, B. Remy, A. Khabbazi, Y. Jannot, B. Baillis, Experimental thermal properties characterization of insulating cork-gypsum composite, Constr.Build. Mater. (2014); 54: 202-209.

2. Y. Elhamdouni, A. Khabbazi, C. Benayad, A. Dadi, O. Idriss Ahmid. Effect of fiber alfa on thermophysical characteristics of a material based on clay. Energy Procedia 74 (2015) 718 $-727$

3. S. Mounir, Y. Maaloufa, A. Cherki, A. Khabbazi, Thermal properties of the composite material clay/granular cork, Constr. Build. Mater.(2014); 70 :183-190.

4. K. El Azhary, Y. Chihab, N. Laaroussi, M. Garoum, M. Mansour. Energy efficiency and thermal properties of the composite material clay-straw. Energy Procedia 00 (2017) 000-000

5. N. Laaroussi, G. Lauriat, M. Garoum, A. Cherki, Y. Jannot, Measurement of thermal properties of brick materials based on clay mixtures, Constr. Build.Mater.(2014); 70:351-361.

6. N. Laaroussi, A. Cherki, M. Garoum, A. Khabbazi, A. Feiz, Thermal properties of a sample prepared using mixtures of clay bricks, Energy Proc.(2013); 42:337-346.
7. Y. Jannot, V. Felix, A. Degiovanni, A centred Hot Plate method for measurement of thermal properties of thin insulating materials, Meas.Sci.Technol. (2010); 21 : 035106.

8. D. Maillet, S. André, J.C.Batsale, A.Degiovanni, C. Moyne. Thermal quadrupoles: solving the heat equation through integral transforms. Chichester (PA): John Wiley \& Sons Ltd; (2000)

9. F.R. De Hoog, An improved method for numerical inversion of Laplace transforms, Society for Industrial and Applied Mathematics, (1982); 3(3): 357-66.

10. D.W. Marquardt, An Algorithm for LeastSquares Estimation of Nonlinear Parameters, J. Soc. Ind. Appl. Math. 11 (1963) 431-441. doi:10.1137/0111030.

11. K. Levenberg, A method for the solution of certain nonlinear problems in least squares. Q. Appl. Math. 2164-8, (1944). 